\title{
Numerical analysis on the current-sharing temperature of LTS/HTS hybrid conductor
}

\author{
CUI YingMin ${ }^{1,2}$, WANG YinShun ${ }^{1 *} \&$ LÜ Gang ${ }^{2}$ \\ ${ }^{1}$ Key Laboratory of HV and EMC Beijing, State Key Laboratory for Alternate Electrical Power System with Renewable Energy Sources, North \\ China Electric Power University, Beijing 102206, China; \\ ${ }^{2}$ Mathematical \& Physical Science School, North China Electric Power University, Baoding 071003, China
}

Received January 26, 2011; accepted April 21, 2012; published online August 6, 2012

\begin{abstract}
The research of LTS/HTS hybrid conductor is a more recent topic in superconducting domain, and the current-sharing temperature is a key parameter to identify the characteristic of superconducting material. In this paper, based on the power-law model, the current-sharing temperatures for LTS and HTS were calculated respectively, and the calculated results have a good agreement with the existing equation results. The current-sharing temperature of a LTS/HTS hybrid superconducting conductor was numerically calculated. The calculated result of hybrid conductor shows that its current-sharing temperature can increase at least $0.2 \mathrm{~K}$ compared with typical LTS conductor with the same normalized transport current, so that the stability of this hybrid conductor is better than typical LTS supercondutor. It indicates that this LTS/HTS hybrid conductor as the substitutes of low-temperature superconductor, will have a wide application prospect and talent commercial value.
\end{abstract}

current-sharing temperature, hybrid conductor, stability

Citation: Cui Y M, Wang Y S, Lü G. Numerical analysis on the current-sharing temperature of LTS/HTS hybrid conductor. Chin Sci Bull, 2012, 57: 3858-3861, doi: $10.1007 / \mathrm{s} 11434-012-5415-5$

To enhance the performance of conventional composite superconductors with large current capacity, a new LTS/HTS hybrid in which HTS is used as a part stabilizer in place of low-resistivity metals, was proposed [1,2]. The performance of every conductor is expressed in terms of current-sharing temperature $\left(T_{\mathrm{CS}}\right)$, which is the key design parameter and the main object of the qualification tests for the ITER, LHD conductors [3]. However current-sharing temperature has not well been studied on the LTS/HTS hybrid conductor.

In this paper, the current-sharing temperature of LTS/ HTS hybrid conductor, consisting of $\mathrm{NbTi} / \mathrm{Cu}$ with Bi2223/ $\mathrm{Ag}$, is numerically calculated based on the power-law model. The calculated current-sharing temperature results of LTS and HTS have been compared with the existing equation results. This paper will provide reference for cryogenic refrigerating and cool conducting technology especially without liquid helium.

*Corresponding author (email: yswang@ @ncepu.edu.cn)

\section{Model}

This kind of LTS/HTS hybrid conductor consists of soldering LTS wire and HTS tape together or by directly winding several LTS wires and HTS tapes together in parallel mode. The LTS $(\mathrm{NbTi} / \mathrm{Cu})$ conductor stabilized with two HTS (Bi2223/Ag) tapes is shown in Figure 1.

Assume that the length of the hybrid conductor in Figure 1 is infinite and the magnetic field is uniform; it is approximately reasonable to neglect the current distribution effects induced by the joints and the couple current effect. We use the model which has been partially tested by experiment [4], where the LTS/HTS hybrid conductor can be considered to be approximately parallel circuit consisting of LTS, HTS, and metal matrix, shown in Figure 2.

As for cooling system and conduction cooled system without liquid helium, adiabatic condition can be approximately adopted. $I_{\mathrm{T}}$ is the total transport current of hybrid conductor; $I_{\mathrm{H}}, I_{\mathrm{L}}$, and $I_{\mathrm{M}}$ are the transport currents through 


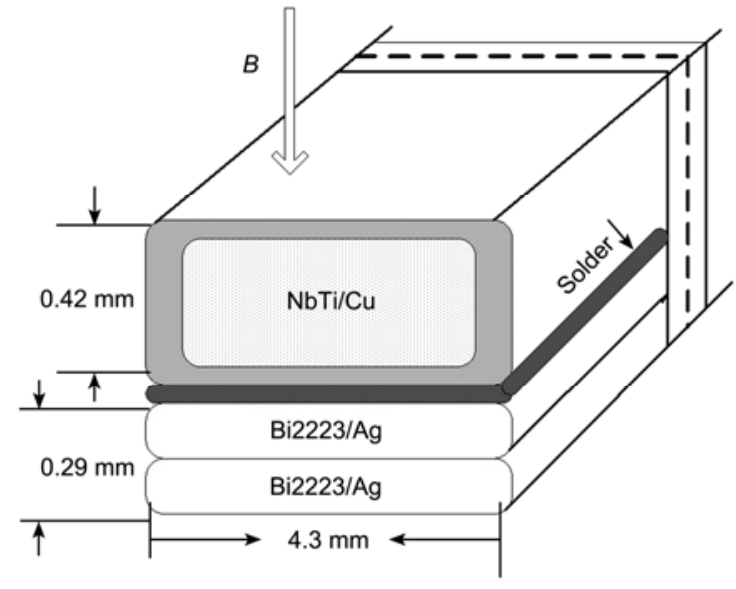

Figure 1 Schematic view of LTS/HTS hybrid conductor with combination of $\mathrm{NbTi} / \mathrm{Cu}$ and $\mathrm{Bi} 2223 / \mathrm{Ag}$ conductors and solders under the magnetic field $B=6 \mathrm{~T}$ (in order to make the critical current of HTS comparable as possible as the LTS, two commercial HTS tapes are selected in simulation).

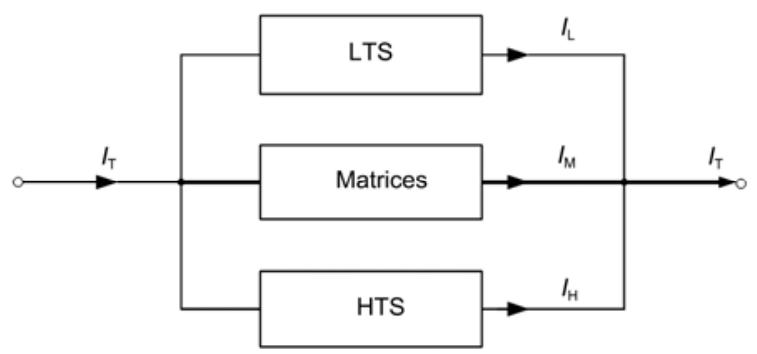

Figure 2 Equivalent parallel circuit of LTS/HTS hybrid conductor.

HTS, LTS, and matrices, respectively. In adiabatic condition, according to the power law model, the above parameters satisfy the following equations [4]:

$$
\left\{\begin{array}{l}
I_{\mathrm{T}}=I_{\mathrm{H}}+I_{\mathrm{L}}+I_{\mathrm{M}}, \\
\left(\frac{I_{\mathrm{H}}}{I_{\mathrm{CH}}}\right)^{n_{\mathrm{H}}}=\left(\frac{I_{\mathrm{L}}}{I_{\mathrm{CL}}}\right)^{n_{\mathrm{L}},}, \\
\left(\frac{I_{\mathrm{H}}}{I_{\mathrm{CH}}}\right)^{15} \times 10^{-4}=\frac{\rho_{\mathrm{avg}}}{S_{\mathrm{M}}} I_{\mathrm{M}},
\end{array}\right.
$$

where $\rho_{\text {avg }}$ is the effective resistivity, and $S_{\mathrm{M}}$ is the crosssections of matrices. $I_{\mathrm{CH}}$ and $I_{\mathrm{CL}}$ are the critical current for HTS and LTS respectively; $n_{\mathrm{H}}$ and $n_{\mathrm{L}}$ are the $n$-value of the power-law for HTS and LTS respectively. The estimation of $\rho_{\text {avg }}$ is given by

$$
\frac{1}{\rho_{\text {avg }}}=\sum_{i=1}^{n} \frac{f_{i}}{\rho_{i}},
$$

where $f_{i}$ and $\rho_{i}$ are volumetric ratio and resistivity of the $i$-th components in matrices except for LTS and HTS. Because the resistivity of superconductors is at least one order of magnitude more than the metal conductor, it is reasonable to neglect.
The critical current of the hybrid conductor is defined as

$$
I_{\mathrm{C}}(T)=I_{\mathrm{CH}}(T)+I_{\mathrm{CL}}(T) .
$$

Under magnetic field of $6 \mathrm{~T}$, dependence of critical currents of $\mathrm{NbTi}$ on temperature can be approximately described by Morgan equation

$$
\begin{aligned}
I_{\mathrm{CL}}(T)= & 959.27\{1-1.195[0.315319(T-4.2) \\
& \left.\left.+0.01528(T-4.2)^{2}-0.00161(T-4.2)^{3}\right]\right\},
\end{aligned}
$$

and the relation of critical current with temperature in two $\mathrm{Bi} 2223 / \mathrm{Ag}$ tapes is approximately described by

$$
I_{\mathrm{CH}}(T)=I_{\mathrm{C}}(0)\left(1-\frac{T}{T_{\mathrm{C}}}\right)^{1.4},
$$

where $I_{\mathrm{C}}(0)=620 \mathrm{~A}, T_{\mathrm{C}}=110 \mathrm{~K}$.

For the sake of convenience, the normalized transport current $\alpha=I_{\mathrm{T}} / I_{\mathrm{C}}$ is defined and used thereafter.

\section{Results and discussion}

Usually there is field $E_{\mathrm{M}}$ along conductor length. Currentsharing temperature is commonly defined as the temperature at which the voltage along the conductor reaches a threshold value defined by the critical electric field of 0.1 $\mu \mathrm{V} / \mathrm{cm}[5,6]$. By solving above equations numerically, the $I_{\mathrm{L}}, I_{\mathrm{H}}$ and $I_{\mathrm{M}}$ are obtained. According to $E_{\mathrm{M}}=I_{\mathrm{M}}{ }^{2} \rho_{\text {avg }} /\left(I_{\mathrm{T}} S_{\mathrm{M}}\right)$ [7], then the field along conductor can be figured out ( $n_{\mathrm{H}}=15, n_{\mathrm{L}}=25$ are used in all the following calculations).

Critical electric fields of LTS, LTS/HTS hybrid, and HTS conductors varied with temperature at $\alpha=0.7$ is shown in Figure 3. It shows that the $T_{\mathrm{CS}}$ for hybrid conductor is between LTS and HTS, and with $0.6 \mathrm{~K}$ larger than LTS when the normalized transport current $\alpha=0.7$.

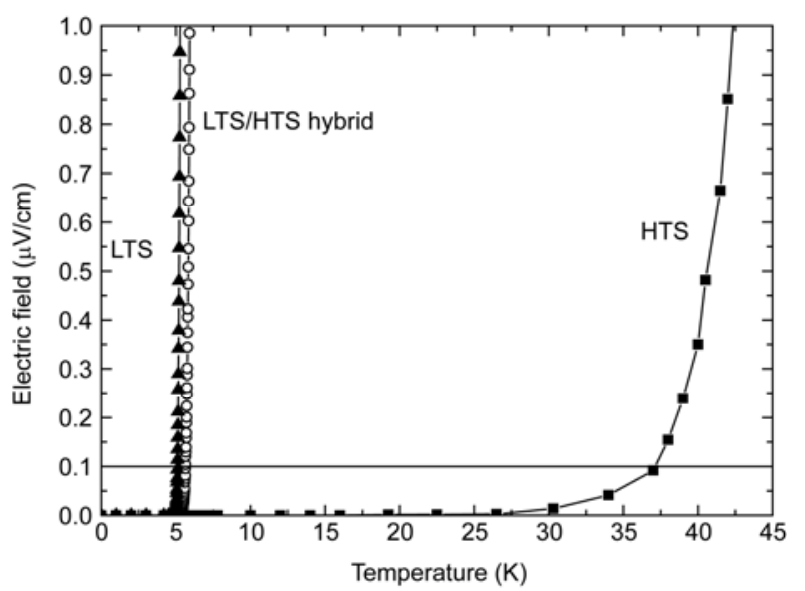

Figure 3 Critical electric fields of LTS, LTS/HTS hybrid, and HTS conductors vs. temperature, normalized transport current $\alpha=0.7$ is used. The $0.1 \mu \mathrm{V} / \mathrm{cm}$ criterion is represented by the solid line, and TCS of LTS, LTS/ HTS hybrid, and HTS conductors are 5.0, 5.6, and 37.1 K respectively. 
Figure 4 shows $T_{\mathrm{CS}}$ comparison of LTS, LTS/HTS hybrid, and HTS conductors. It shows that $T_{\mathrm{CS}}$ for LTS/HTS hybrid are between LTS and HTS. While the normalized transport current $\alpha<0.4, T_{\mathrm{CS}}$ for hybrid conductor approaches to HTS one; while $\alpha>0.4$, it approaches to LTS one and with a few $\mathrm{K}$ larger than LTS. There is a turning point at about $\alpha=0.4$, because the total transport current at this point reaches the critical current of the hybrid's HTS part and leads to this part's quench. Thus, while $\alpha>0.4$, the current-sharing temperature of hybrid conductor is close to the typical LTS and with at least $0.2 \mathrm{~K}$ larger than that of LTS at the same circumstance (see Table 1).

$T_{\mathrm{CS}}$ can also be estimated by [8]

$$
T_{\mathrm{CS}}=T_{\mathrm{C}}-\frac{I_{\text {op }}}{I_{\mathrm{C}}\left(T_{\mathrm{op}}\right)}\left(T_{\mathrm{C}}-T_{\mathrm{op}}\right),
$$

where $T_{\mathrm{op}}$ and $T_{\mathrm{C}}$ are operating temperature and critical temperature respectively; $I_{\mathrm{op}}$ and $I_{\mathrm{C}}\left(T_{\mathrm{op}}\right)$ are current and critical current at operating temperature respectively. Figure 5 shows the $T_{\mathrm{CS}}$ comparisons of the numerically calculated result and the equation result for LTS and HTS separately. The results show that the calculated result accords with the equation result.

\section{Conclusions}

The current-sharing temperature of LTS/HTS hybrid conductor in which high-temperature superconductor is used as stabilizers has been numerically investigated. The result shows that the $T_{\mathrm{CS}}$ of this hybrid conductor is between typical LTS and HTS ones. When the total transport current of hybrid conductor is over $40 \%$ of its critical current, its current-sharing temperature will be more close to typical LTS and with at least $0.2 \mathrm{~K}$ larger than that of LTS with the same normalized transport current. The LTS/HTS hybrid conductor, as a possible substitute material on LTS with pure aluminum, is to work in the low temperature like LTS. For large cooling and conducting cooled system without liquid

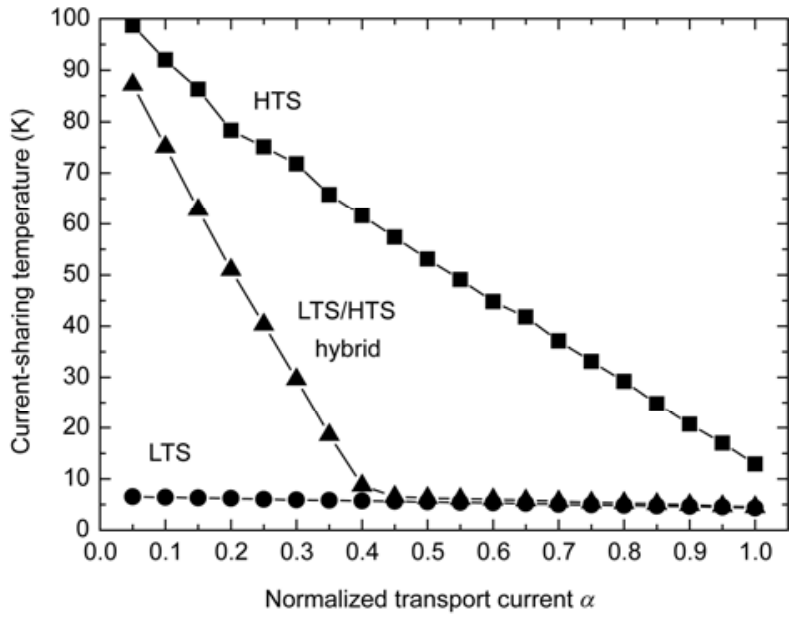

Figure 4 Calculated $T_{\mathrm{CS}}$ comparison for LTS, LTS/HTS hybrid, and HTS, conductors.

Table 1 Comparison of current-sharing temperature for LTS and LTS/HTS hybrid conductors

\begin{tabular}{ccc}
\hline $\begin{array}{c}\text { Normalized transport } \\
\text { current } \alpha\end{array}$ & LTS $T_{\mathrm{CS}}(\mathrm{K})$ & $\begin{array}{c}\text { LTS/HTS hybrid } \\
\text { conductor } T_{\mathrm{CS}}(\mathrm{K})\end{array}$ \\
\hline 0.1 & 6.4 & 75.1 \\
0.2 & 6.2 & 51.0 \\
0.3 & 5.9 & 29.6 \\
0.4 & 5.7 & 8.7 \\
0.5 & 5.6 & 6.3 \\
0.6 & 5.3 & 6.0 \\
0.7 & 5.0 & 5.6 \\
0.8 & 4.8 & 5.3 \\
0.9 & 4.6 & 4.9 \\
1.0 & 4.3 & 4.5 \\
\hline
\end{tabular}

helium, the temperature reduction of $\sim \mathrm{mK}$ means large numbers of energy consumption in this extremely low temperature. Moreover, the working temperature range for these systems without liquid helium is very small, usually within several hundreds of $\mathrm{mK}$, so the superconducting magnets
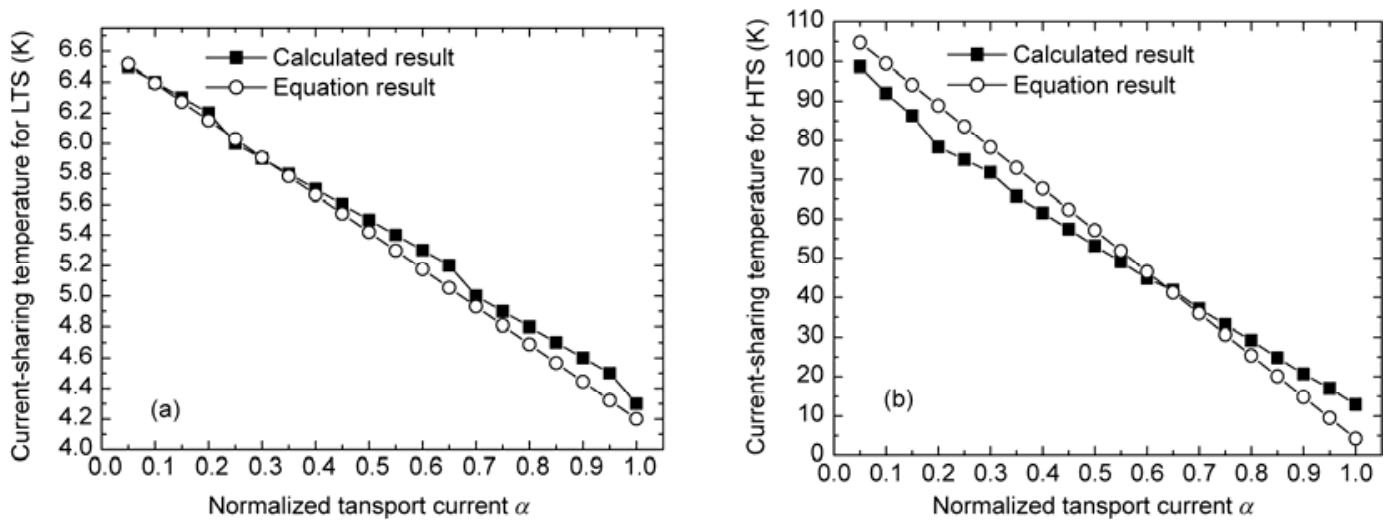

Figure $5 T_{\mathrm{CS}}$ difference between the calculated result and the equation result for LTS (a) and HTS (b). 
cooled by a cryogenic refrigerator without liquid helium usually have worse cryogenic stability than the system with the liquid helium. Therefore, a few K's increase of $T_{\mathrm{CS}}$ compared with typical LTS made us believe that this LTS/ HTS hybrid conductor will be explored a new research field for the cryogenic stability of low temperature superconductors. Experiment of current-sharing temperature for LTS/HTS hybrid conductor will be undertaken in our future work.

We thank Dr. Pi Wei for the helpful calculation work. This work was supported by the National Natural Science Foundation of China (51077051), the Specialized Research Fund for the Doctoral Program of Higher Education (D00033) and the Doctoral Research Fund of North China Electric Power University (10QL27).

1 Wang Y S, Zhao X, Han J J, et al. A new type of hybrid structure superconductor wires and tapes. Patent No. 200410048208.8, China

2 Bansal G, Yanagi N, Hemmi T, et al. Stability measurements of LTS/ HTS hybrid superconductor. Fusion Eng Des, 2006, 81: 2485-2489

3 Bagnasco M. Calorimetric method for current sharing temperature measurements in ITER conductor samples in SULTAN. Fusion Eng Des, 2009, 84: 423-426

4 Guan X J, Wang Y S, Zhou W W. Current distribution and stability of LTS/HTS hybrid superconducting conductors. Sci China Tech Sci, 2010, 53: 2000-2007

5 Breschi M, Ribani P L, Bellina F. Electromagnetic analysis of the voltage-temperature characteristics of the ITER TF conductor samples. IEEE Trans Appl Supercond, 2009, 19: 1512-1515

6 Heller R, Ciazynski D, Duchateau J L, et al. Evaluation of the current sharing temperature of the ITER toroidal field model coil. IEEE Trans Appl Supercond, 2003, 13: 1447-1451

7 Takahashi Y, Isono T, Koizumi N, et al. Performance of Japanese $\mathrm{Nb}_{3} \mathrm{Sn}$ conductors for ITER toroidal field coils. IEEE Trans Appl Supercond, 2008, 18: 471-473

8 Iwasa Y. Case Studies in Superconducting Magnets Design and Operational Issues. New York: Plenum Press, 1994. 2

Open Access This article is distributed under the terms of the Creative Commons Attribution License which permits any use, distribution, and reproduction in any medium, provided the original author(s) and source are credited. 